\title{
Características de los tratamientos biológicos en enfermedades reumáticas en Argentina: quinto informe del registro BIOBADASAR
}

\author{
M. de la Vega, G. Casado, J. Roberti, M.G. Gómez, A. Benitez, C. Battagliotti, I. Exeni, C. Gobbi, P. Astesana, R. Quintana, \\ A. Bertoli, I. Strusberg, S. Paira, E. Mussano, D. Vidal, M. Rama, E. Cavillon, A. Quinteros, A. Capuccio, G. Bovea, M. \\ de la Sota, M. Larroudé, A. Granel, A. Marcos, A. Smichowski, F. Tamaño, B. Barrios, J.M. Bande, E. Soriano, A. Eimon, \\ A. Alvarez, G. Gomez, S. Agüero, M. Sacnun, M. Garcia, C. Costi, S. Soares de Souza, E. Velozo, M. Diaz, J. Sarano, \\ B. Pons-Estel, V. Saurit, E. Miretti, D. Dubinsky, A. Perez Davila, A. Bohr, M. Mamani, S. Scarafia C. Sanchez Andia, K. \\ Kirmayr, J.C. Barreira, G. Citera \\ Centros Participantes \\ Ciudad de Buenos Aires: CEIM Investigaciones Médicas, Hospital C. Milstein, Consultorio Dra. Larroudé, Hospital Tornú, Hospital Italiano, \\ Instituto de Rehabilitación Psicofísica, Hospital de Clínicas, Hospital Rivadavia, CEMIC, Hospital Penna, Instituto Lanari, Atención Integral \\ en Reumatología, REUMAR, Hospital Británico, Hospital de Rehabilitación Rocca. \\ Córdoba: Sanatorio Parque, Sanatorio Allende, Instituto Strusberg, Hospital Privado, Hospital de Niños, Hospital Nacional de Clínicas, \\ Consultorio Dra. Cavillon. \\ Tucumán: Centro Integral Reumatológico. \\ Santa Fe: Hospital de Niños Dr. O. Alassia, Sanatorio Parque, Hospital Cullen, Hospital Provincial. \\ Buenos Aires: Consultorio Dra. de la Sota, Centro Platense de Reumatología, Hospital San Martín. \\ Catamarca: Sanatorio Pasteur. \\ Río Negro: Centro Bariloche. \\ Entre Ríos: Consultorio Dr. Caprarulo, Sanatorio Adventista del Plata.
}

\section{Resumen}

Introducción: El proyecto BIOBADASAR (Registro argentino de eventos adversos con tratamientos biológicos en reumatología) comenzó en agosto de 2010, para recabar información a largo plazo sobre los eventos adversos en tratamientos biológicos en pacientes con enfermedades reumáticas en la práctica clínica cotidiana en Argentina.

Pacientes y método: Se registraron datos de cada paciente, tratamientos y acontecimientos adversos relevantes o importantes. Los pacientes debían tener enfermedad diagnosticada y tratada con un agente biológico. Cada caso se comparó con un control: un paciente con tratamiento no biológico con características demográficas similares. Se analizaron los datos con análisis de la varianza, con test de $t$ de Student, Mann Whitney, test chi2, o test exacto de Fisher. El análisis de supervivencia de los tratamientos hasta su discontinuación o interrupción se realizó con el método de Kaplan-Meier y test log-rank.

Resultados: Se incluyeron 1888 pacientes con agentes biológicos $(58,7 \%)$ y 1326 con drogas no biológicas $(41,26 \%)$ al ingresar al

\section{Abstract}

Background: BIOBADASAR (Argentine Registry of Adverse Events in Biological Treatments in Rheumatology) was started in August 2010 to obtain long-term information of patients with rheumatic diseases, treatments and adverse events in everyday clinical practice. Patients and methods: Data on patients' demographics, treatments and adverse events were collected. Patients had a diagnosis of a rheumatic disease and were treated with biological agent. To compare information, a control group was included, consisting of patients treated with similar demographic characteristics but treated with a non-biological agent. Data were analysed with Anova, Student's t, Mann Whitney, chi2, Fisher's exact tests, as appropriate. Survival analysis of treatments was performed with Kaplan-Meier curves and log-rank test.

Results: 3214 patients were included, 1888 (58.7\%) initially treated with biological agents and $1326(41.26 \%)$ with non-biological agents. Mean age at time of diagnosis $41.6 \pm 15.4$ vs. $45.3 \pm 16.3$ years, biological vs. non-biological groups respectively, $p<0.05$. Main diagnosis: rheumatoid arthritis $(n=2502,77.8 \%)$, psoriatic ar- 
registro. Los diagnósticos principales fueron artritis reumatoidea $(\mathrm{n}=2502,77,8 \%)$ y artritis psoriásica $(\mathrm{n}=266,8,3 \%)$. Se analizaron $2720(60,7 \%)$ tratamientos biológicos vs. $1759(39,3 \%)$ tratamientos no biológicos. Los agentes biológicos más utilizados fueron etanercept $(25,1 \%)$ y adalimumab $(13,1 \%)$. La supervivencia de los tratamientos no biológicos fue mayor que la de los agentes biológicos. Las principales causas de discontinuación fueron la ineficacia y eventos adversos. Se informaron 1902/2434 (78,1\%) acontecimientos adversos en el grupo biológicos y 532/2434 $(21,9 \%)$ en el grupo no biológico. El 39\% fue algún tipo de infección; el $7,3 \%$, trastornos de la piel; el $5,8 \%$, trastornos de la sangre, y el $5,8 \%$, trastornos gastrointestinales.

Conclusiones: En la clínica, los eventos adversos no difieren significativamente de los resultados de otros registros. Se han implementado mecanismos más estrictos de monitorización para mejorar la calidad de los datos, de los informes, y para que, al saber sobre la realidad de los pacientes, se mejore su atención.

Palabras clave: BIOBADASAR, registros, biológicos, eventos adversos. thritis ( $n=266,8.3 \%$ ). 2720 (60.7\%) biological treatments vs. 1759 $(39.3 \%)$ non-biological were registered. Most frequent biological drugs used were etanercept (25.1\%), adalimumab (13.1\%). Survival of non-biological treatments was higher than survival of treatment with biological agents. Main causes for treatment withdrawal were lack of efficacy and adverse events. A total of 1902/2434 (78.1\%) adverse events were reported in the biological treatment group vs. $532 / 2434(21.9 \%)$ in the non-biological treatment group. Thirtynine percent of adverse events were some type of infection, $7.3 \%$ affected the skin, $5.8 \%$ were blood conditions, and $5.8 \%$ were gastrointestinal complications.

Conclusions: This is the fifth registry report. In everyday clinical practice, adverse events are not significantly different from those reported in other registries. Rigorous monitoring could improve data and reporting quality to achieve patients' better care.

Key words: BIOBADASAR, registries, biologics, adverse events.

\section{Introducción}

El tratamiento con drogas biológicas de enfermedades reumáticas ha logrado un mejor pronóstico y la remisión de la enfermedad en un porcentaje importante de pacientes ${ }^{1}$. El proyecto BIOBADASAR (Registro argentino de eventos adversos con tratamientos biológicos en reumatología) se fundamenta en la necesidad de contar con datos sobre tratamiento con medicamentos biológicos para pacientes con enfermedades reumáticas en el contexto de la práctica clínica cotidiana en Argentina. Existen experiencias exitosas de registros en otros países, especialmente cuando se planifican y se implementan desde un organismo central o una sociedad científica; en el caso argentino, la Sociedad Argentina de Reumatología. Asimismo, las investigaciones observacionales como los registros aportan información sobre efectos a largo plazo de los tratamientos y en poblaciones con mayor diversidad que la de los ensayos clínicos controlados y aleatorizados. De este modo, el registro de la Sociedad Española de Reumatología, BIOBADASER, brindó información sobre la incidencia de tuberculosis en pacientes tratados con agentes biológicos antes de las recomendaciones de profilaxis $^{2}$ o sobre la mortalidad en pacientes tratados con anti-TNF ${ }^{3}$. El registro de la Sociedad Británica de Reumatología (BSRBR) proporcionó información sobre el riesgo relativo de infecciones, incidencia de psoriasis y de cáncer en pacientes tratados con anti-TNFs ${ }^{4-6}$. A partir del registro sueco, ARTIS, y del alemán, RABBIT, se obtuvo información sobre riesgo de tumores y de herpes zóster en el tratamiento con agentes biológicos, respectivamente ${ }^{7,8}$. Otros registros similares son los de Noruega, Suiza y Estados Unidos ${ }^{9-11}$. El objetivo del presente trabajo es comunicar los resultados del registro BIOBADASAR sobre los tratamientos con agentes biológicos en enfermedades reumáticas y los acontecimientos adversos, cinco años después de haber incorporado los primeros pacientes.

\section{Métodos}

BIOBADASAR es un registro de acontecimientos adversos, tomando como base metodológica el registro español BIOBADASER, para determinar la seguridad de las terapias biológicas en enfermedades reumáticas. Se registran los datos de los pacientes (sexo, edad, diagnóstico, 
fecha de diagnóstico), de los tratamientos (tipo, fecha de inicio y fin de tratamiento) y de los acontecimientos adversos (fecha, descripción). Los pacientes incluidos deben tener cualquier enfermedad diagnosticada tratada por reumatólogos, para la que se haya utilizado un tratamiento de origen biotecnológico; deben haber iniciado tratamiento con dicha terapia biológica en un centro participante; pueden haber suspendido la terapia biológica por cualquier causa, siempre y cuando no haya transcurrido más de un año desde que se aplicara el tratamiento y que se disponga de todos los datos necesarios para el registro (del paciente, del tratamiento y de los acontecimientos adversos). Los pacientes deben autorizar la recopilación prospectiva de datos según fórmula indicada en consentimiento informado. Por cada paciente con tratamiento con agente biológico, se incorpora al registro un paciente con tratamiento no biológico con características similares a las del paciente en tratamiento biológico en cuanto a sexo, edad y tiempo de evolución. En el registro se recogen datos de acontecimientos adversos que produzca la muerte, ponga en peligro la vida, precise de ingreso hospitalario o lo prolongue, produzca una discapacidad persistente o importante o produzca malformaciones congénitas o que, sin producir la muerte ni poner en peligro inmediato la vida del paciente, comprometa al paciente, su calidad de vida, o requieran intervención para prevenir alguno de los resultados enumerados en la definición anterior. Se realizó una monitorización on-line permanente de los datos ingresados por los centros. Se incluyeron los datos de pacientes de 52 centros de reumatología de Argentina, ingresados desde el $1^{\circ}$ agosto de 2010 al 20 de agosto de 2015.

Se describieron los resultados de los análisis descriptivos de las variables en medias, desvío estándar, medianas con rangos, frecuencias y porcentajes, según corresponda. Las diferencias entre los grupos se realizaron con análisis de la varianza, con test de t de Student, Mann Whitney, según corresponda. Las proporciones se compararon con los test chi2 o test exacto de Fisher, según corresponda. El análisis de supervivencia se realizó tomando en cuenta la supervivencia de los tratamientos hasta su discontinuación o interrupción. Las curvas de supervivencia se realizaron con el método Kaplan-Meier y las diferencias entre ellas se evaluaron con test log-rank. Se evaluaron los riesgos relativos para categorías de acontecimientos adversos. El nivel de significancia estadística se estableció en $p<0,05$. Se utilizó el programa STATA v11 (College Station, TX, USA).

\section{Resultados}

Se analizaron los datos provenientes de 34 centros públicos y privados de todo el país correspondientes a 3214 pacientes,

\begin{tabular}{|c|c|c|c|}
\hline Características y diagnósticos & Pacientes en tratamiento biológico & Pacientes en tratamiento no biológico & $\begin{array}{c}\text { Total } \\
n=3214\end{array}$ \\
\hline Edad al diagnóstico,años, media $\pm D E$ & $41,6 \pm 15,4$ & $45,3 \pm 16,3$ & $43,1 \pm 15,9^{\star}$ \\
\hline Mujeres, n (\%) & $1470(77,9)$ & $1064(80,2)$ & $2534(78,8)$ \\
\hline Artritis idiopática juvenil, n (\%) & $67(3,5)$ & $41(3,1)$ & $108(3,4)$ \\
\hline Artritis reactiva, $\mathrm{n}(\%)$ & $4(0,2)$ & $5(0,4)$ & $9(0,3)$ \\
\hline Artritis reumatoide, $\mathrm{n}(\%)$ & $1437(76,1)$ & $1065(80,3)$ & $2502(77,8)^{\star}$ \\
\hline Artritis o Spa psoriásica, n (\%) & $184(9,7)$ & $82(6,2)$ & $266(8,3)^{\star}$ \\
\hline Enfermedad Behcet, n (\%) & $6(0,3)$ & $2(0,2)$ & $8(0,2)$ \\
\hline Enfermedad de Still, n (\%) & $7(0,4)$ & $0(0,0)$ & $7(0,2)$ \\
\hline Esclerodermia, n (\%) & $7(0,4)$ & $10(0,8)$ & $17(0,5)$ \\
\hline Espondilitis anquilosante, $\mathrm{n}(\%)$ & $53(2,8)$ & $21(1,6)$ & $74(2,3)^{\star}$ \\
\hline Espondiloartropatía indiferenciada, n (\%) & $15(0,8)$ & $10(0,8)$ & $25(0,8)$ \\
\hline Lupus eritematoso sistémico, n (\%) & $54(2,9)$ & $56(4,2)$ & $110(3,4)^{\star}$ \\
\hline Poliartritis crónica seronegativa, n (\%) & $6(0,3)$ & $3(0,2)$ & $9(0,3)$ \\
\hline Polimiositis/Dermatomiositis, n (\%) & $12(0,6)$ & $8(0,6)$ & $20(0,6)$ \\
\hline Uveítis sin enfermedad reumática, n (\%) & $5(0,3)$ & $1(0,1)$ & $6(0,2)$ \\
\hline Vasculitis, n (\%) & $13(0,7)$ & $11(0,8)$ & $24(0,7)$ \\
\hline Otros diagnósticos, n (\%) & $18(1,0)$ & $11(0,8)$ & $29(0,9)$ \\
\hline Total, n (\%) & $1888(100)$ & $1326(100)$ & $3214(100)$ \\
\hline
\end{tabular}

Tabla 1. Características y diagnósticos según grupo de tratamiento. 
de los cuales 1888 (58,7\%) pacientes fueron tratados con agentes biológicos (grupo casos) y $1326(41,26 \%$ ) pacientes fueron tratados con drogas no biológicas (grupo controles) al momento de ingresar al registro. La edad promedio al momento del diagnóstico fue de 41,6 $\pm 15,4$ años en el grupo de pacientes con agentes biológicos y 45,3 $\pm 16,3$ años para el grupo control $(p<0,05)$. En el grupo total, 2534 (78,8\%) fueron mujeres, sin diferencia estadísticamente significativa según el tipo de tratamiento (Tabla 1).

Los diagnósticos principales fueron artritis reumatoidea $(\mathrm{n}=2502 / 3214,77,8 \%)$, artritis psoriásica $(\mathrm{n}=266 / 3214$, $8,3 \%)$, artritis idiopática juvenil $(\mathrm{n}=108 / 3214,3,4 \%)$ y lupus eritematoso sistémico $(\mathrm{n}=110 / 3214,3,4 \%)$ (Tabla 1). Las comorbilidades presentes antes del comienzo del tratamiento más frecuentemente informadas fueron la hipertensión ( $\mathrm{n}=732 / 3214,22,8 \%)$, la osteoporosis $(n=467 / 3214,14,6 \%)$ y la hipercolesterolemia $(n=392 / 3214$, $12,2 \%)$. Sólo en el caso de la insuficiencia cardíaca se observó una diferencia estadísticamente significativa entre los grupos de tratamiento (Tabla 2). Se informaron $47(1,46 \%)$ muertes, $38 / 1888(2,0 \%)$ y $9 / 1326(0,6 \%)$ en el grupo de tratamiento biológico y no biológico, respectivamente, $\mathrm{p}<0,05$; en 15 casos, se atribuyó la muerte al acontecimiento adverso.

\begin{tabular}{|l|c|c|c|}
\hline Comorbilidades & $\begin{array}{c}\text { Pacientes en } \\
\text { tratamiento } \\
\text { biológico }\end{array}$ & $\begin{array}{c}\text { Pacientes en } \\
\text { tratamiento } \\
\text { no biológico }\end{array}$ & Total \\
\hline Cáncer, $\mathrm{n}(\%)$ & $34(1,8)$ & $26(2)$ & $60(1,9)$ \\
\hline Linfoma, $\mathrm{n}(\%)$ & $2(0,1)$ & $1(0,1)$ & $3(0,1)$ \\
\hline Cardiopatía isquémica, $\mathrm{n}(\%)$ & $33(1,7)$ & $19(1,4)$ & $52(1,6)$ \\
\hline Diabetes, $\mathrm{n}(\%)$ & $127(6,7)$ & $73(5,5)$ & $200(6,2)$ \\
\hline Fumador, $\mathrm{n}(\%)$ & $178(9,4)$ & $99(7,5)$ & $277(8,6)$ \\
\hline Insuficiencia renal, $\mathrm{n}(\%)$ & $18(0,9)$ & $12(0,9)$ & $30(0,9)$ \\
\hline Insuficiencia cardíaca, $\mathrm{n}(\%)$ & $21(1,1)$ & $6(0,4)$ & $27(0,8)^{\star}$ \\
\hline Hipercolesterolemia, $\mathrm{n}(\%)$ & $242(12,8)$ & $151(11,4)$ & $392(12,2)$ \\
\hline EPOC, $\mathrm{n}(\%)$ & $42(2,2)$ & $17(1,3)$ & $59(1,8)$ \\
\hline Hipertensión arterial, $\mathrm{n}(\%)$ & $439(23,2)$ & $293(22,1)$ & $732(22,8)$ \\
\hline Osteoporosis, $\mathrm{n}(\%)$ & $288(15,2)$ & $179(13,5)$ & $467(14,5)$ \\
\hline Virus Epstein Barr, $\mathrm{n}(\%)$ & $7(0,4)$ & $3(0,2)$ & $10(0,31)$ \\
\hline Virus hepatitis $\mathrm{B}, \mathrm{n}(\%)$ & $13(0,7)$ & $10(0,7)$ & $23(0,7)$ \\
\hline HBAgs+, $\mathrm{n}(\%)$ & $5(0,3)$ & $7(0,5)$ & $12(0,37)$ \\
\hline Virus hepatitis C, $\mathrm{n}(\%)$ & $8(0,4)$ & $2(0,1)$ & $10(0,3)$ \\
\hline
\end{tabular}

Abreviaturas: EPOC, enfermedad pulmonar obstructiva crónica; HBAg, antígeno hepatitis $B$. ${ }^{*} p<0,05$.

\section{Tabla 2. Comorbilidades diagnosticadas.}

Se analizaron 4479 ciclos de tratamientos de los cuales $2720 / 4479(60,7 \%)$ fueron con agentes biológicos y $1759 / 4479(39,3 \%)$ con tratamientos no biológicos. En el grupo de agentes biológicos, los más utilizados fueron etanercept $(\mathrm{n}=1125,25,1 \%)$, adalimumab $(\mathrm{n}=588,13,1 \%)$, abatacept $(n=271,6 \%)$ y rituximab $(n=221,4,9 \%)$ (Tabla 3). La gravedad de la enfermedad al inicio del tratamiento informada según DAS28 fue significativamente más alta en el grupo de tratamiento biológico comparado con el grupo no biológico: $5,4 \pm 1,4$ vs. $4,7 \pm 1,3$, respectivamente, $\mathrm{p}<0,05$.

\begin{tabular}{|l|c|}
\hline Fármaco & Frecuencia (porcentaje) \\
\hline Droga no biológica, $\mathrm{n}(\%)$ & $1759(39,27)$ \\
\hline Etanercept, $\mathrm{n}(\%)$ & $1125(25,12)$ \\
\hline Adalimumab, $\mathrm{n}(\%)$ & $588(13,13)$ \\
\hline Abatacept, $\mathrm{n}(\%)$ & $271(6,05)$ \\
\hline Rituximab, $\mathrm{n}(\%)$ & $221(4,93)$ \\
\hline Infliximab, $\mathrm{n}(\%)$ & $168(3,75)$ \\
\hline Tocilizumab, $\mathrm{n}(\%)$ & $127(2,84)$ \\
\hline Certolizumab, $\mathrm{n}(\%)$ & $102(2,28)$ \\
\hline Golimumab, $\mathrm{n}(\%)$ & $49(1,09)$ \\
\hline Tofacitinib, $\mathrm{n}(\%)$ & $45(1,00)$ \\
\hline Belimumab, $\mathrm{n}(\%)$ & $21(0,47)$ \\
\hline Ustekinumab, $\mathrm{n}(\%)$ & $2(0,04)$ \\
\hline Anakinra, $\mathrm{n}(\%)$ & $1(0,02)$ \\
\hline
\end{tabular}

Tabla 3. Fármacos utilizados en los tratamientos de enfermedades reumáticas.

La discontinuación se produjo en 1467 (32,8\%) de los 4479 ciclos de tratamiento y fue $41,1 \%(n=1119 / 2720)$ para los tratamientos con agentes biológicos y 19,8\% $(\mathrm{n}=348 / 1759)$ para los controles, $\mathrm{p}<0,05$. Entre los tratamientos discontinuados, las principales causas fueron la ineficacia en 520/1467 (35,5\%) tratamientos, 417/1119 $(37,3 \%)$ en el grupo biológico vs. $103 / 348(29,6 \%)$ en el grupo control, $(\mathrm{p}<0,05)$ y eventos adversos $455 / 1467$ $(31,0 \%)$, con $357(31,9 \%)$ en el grupo biológico vs. $98(28,2 \%)$, diferencia no significativa; en el grupo de tratamiento no biológico se observó un mayor porcentaje de discontinuación por pérdida de paciente y por remisión de la enfermedad (Tabla 4). La supervivencia de los tratamientos no biológicos fue significativamente mayor que la de los tratamientos con los agentes biológicos (Figura 1). El cociente de riesgo (hazard ratio) de discontinuación de tratamiento por cualquier motivo en el grupo de medicamento biológico comparado con no biológico fue 3,18 (IC 95\% 2,81-3,61).

Se estudiaron 2434 acontecimientos adversos; 1902/2434 (78,1\%) acontecimientos adversos se informaron en el grupo tratado con agentes biológicos y $532 / 2434(21,9 \%)$ en el grupo tratado con medicación 


\begin{tabular}{|l|c|c|c|}
\hline Motivo & $\begin{array}{c}\text { Tratamientos } \\
\text { biológicos }\end{array}$ & $\begin{array}{c}\text { Tratamientos } \\
\text { no biológicos }\end{array}$ & Total \\
\hline $\begin{array}{l}\text { Total tratamientos } \\
\text { suspendidos, } \mathrm{n}(\%)\end{array}$ & $1119(41,1)$ & $348(19,8)$ & $1467(45,6)^{\star}$ \\
\hline $\begin{array}{l}\text { Acontecimiento } \\
\text { adverso, } \mathrm{n}(\%)\end{array}$ & $357(31,9)$ & $98(28,2)$ & $455(31,02)$ \\
\hline Desconocido, $\mathrm{n}(\%)$ & $15(1,3)$ & $4(1,2)$ & $19(1,30)$ \\
\hline $\begin{array}{l}\text { Embarazo 0 deseo } \\
\text { gestacional, } \mathrm{n}(\%)\end{array}$ & $17(1,5)$ & $7(2,0)$ & $24(1,64)$ \\
\hline Ineficacia, $\mathrm{n}(\%)$ & $417(37,3)$ & $103(29,6)$ & $520(35,45)^{\star}$ \\
\hline Otros, $\mathrm{n}(\%)$ & $228(20,4)$ & $58(16,7)$ & $286(19,50)$ \\
\hline Pérdida de paciente, $\mathrm{n}(\%)$ & $65(5,8)$ & $59(17,0)$ & $124(8,45)^{\star}$ \\
\hline Remisión, $\mathrm{n}(\%)$ & $20(1,8)$ & $19(5,5)$ & $39(2,66)^{\star}$ \\
\hline * $\mathrm{p}<0,05$. & & & \\
\hline
\end{tabular}

Tabla 4. Motivo por los que se suspendió o interrumpió tratamiento.

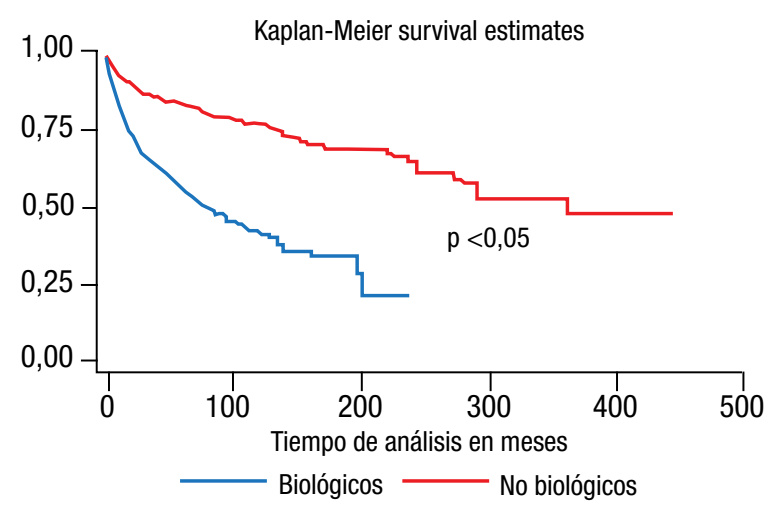

Nota: Se encontró una diferencia estadísticamente significativa entre la supervivencia del tratamiento no biológico y biológico.

Figura 1. Curvas de supervivencia de tratamientos.

no biológica. Del total de acontecimientos adversos, $2094 / 2434(86,0 \%)$ fueron informados como no graves, $268 / 2434(11,0 \%)$ casos fueron graves, con una diferencia estadísticamente significativa entre los dos grupos; 232 $1268(86,6 \%)$ vs. $36 / 268(13,4 \%)$ en grupo con agentes biológicos vs. no biológico, respectivamente, $\mathrm{p}<0,05$. Se informó que 44/2434 (1,8\%) acontecimientos adversos tuvieron desenlace mortal, 144/2434 (5,9\%) tuvieron recuperación con alguna secuela mientras que 1879/2434 $(77,2 \%)$ casos tuvieron como desenlace la recuperación sin secuelas; en este último tipo de desenlace, se observó una diferencia entre el grupo tratado con biológico vs. en el grupo tratado con no biológico: 1571/1879 (80,7\%) vs. $362 / 1879(19,3 \%)$. Con respecto a la clasificación de los acontecimientos adversos, 948 (39\%) fueron algún tipo de infección, 178 (7,3\%) fueron trastornos de la piel, 142
$(5,8 \%)$ fueron trastornos de la sangre, y $142(5,8 \%)$ fueron trastornos gastrointestinales (Tabla 5). Dentro de cada una de estas categorías, los acontecimientos más comunes fueron los siguientes: en infecciones, las más comunes fueron informadas como infecciones cutáneas, del tracto respiratorio superior y bronquitis, con $147(15,4 \%)$, $144(15,1 \%)$ y $129(13,6 \%)$ casos, respectivamente; en trastornos de la piel, lo más frecuente fue la lesión de piel en $110(61,8 \%)$ de los casos; en trastornos de la sangre hubo $62(43 \%)$ casos de alteración en médula ósea, y en trastornos gastrointestinales se informó que $49(34,5 \%)$ casos fueron infecciones gastrointestinales y 18 (12,7\%) de estos eventos fueron dolores abdominales. En la Tabla 5 se muestran los riesgos relativos de eventos adversos para el tratamiento con agentes biológicos de acuerdo a lo informado en el registro.

\section{Discusión}

Se presenta el informe preliminar del proyecto BIOBADASAR que evalúa acontecimientos adversos, patrones de uso y supervivencia de los tratamientos para enfermedades reumáticas en la práctica clínica cotidiana en Argentina. A diferencia de lo que ocurre en otros registros similares, en el registro argentino se ingresan también pacientes tratados con medicación no biológica; no obstante, el grupo de tratamiento con agentes biológicos continúa siendo mayor.

Al igual de lo que se ve en otros registros, la gran mayoría de los pacientes tienen un diagnóstico de artritis reumatoidea ${ }^{11,12}$, con un porcentaje mayor que lo que se ve en otros registros. La comorbilidad informada al momento del inicio del tratamiento más común fue la hipertensión arterial. Si bien las proporciones son pequeñas, la insuficiencia cardíaca fue más común en el grupo de pacientes tratados con agentes biológicos, la cual no constituye una contraindicación absoluta para este tipo de tratamiento pero se desaconseja en pacientes con insuficiencia cardíaca crónica avanzada NYHA clases II y IV ${ }^{1}$.

En este registro, el agente biológico más utilizado fue el etanercept, seguido por el adalimumab y abatacept; mientras que en el registro brasileño, fue el infliximab ${ }^{12}$. Esta diferencia podría deberse a los tiempos de aprobación de cada agente en cada país. La principal causa de discontinuación de tratamientos continúa siendo la ineficacia o pérdida de eficacia del fármaco, al igual que sucede en los registros de Brasil y España ${ }^{12,13}$. Esto quizás tenga relación con fenómenos inherentes a la 


\begin{tabular}{|c|c|c|c|c|}
\hline Acontecimiento adverso & $\begin{array}{c}\text { Tratamiento biológico } \\
2720\end{array}$ & $\begin{array}{c}\text { Tratamiento no biológico } \\
1759\end{array}$ & Total & $\begin{array}{l}\text { Riesgo Relativo (Risk ratio) } \\
\text { en tratamiento biológico } \\
\text { (Intervalo confianza 95\%) }\end{array}$ \\
\hline Infecciones, n (\%) & $806(42,4)$ & $142(26,7)$ & $948(39,0)$ & $3,67(3,10-4,34)^{\star}$ \\
\hline Lesiones traumáticas, n (\%) & $51(2,7)$ & $25(4,7)$ & $76(3,1)$ & $1,32(0,82-2,12)$ \\
\hline Neoplasias, n (\%) & $65(3,2)$ & $23(4,3)$ & $88(3,6)$ & $1,82(1,14-2,93)^{\star}$ \\
\hline T. cardíacos, n (\%) & $40(2,1)$ & $6(1,1)$ & $46(1,9)$ & $4,33(1,84-10,19)^{\star}$ \\
\hline T. de la piel, $\mathrm{n}(\%)$ & $156(8,2)$ & $22(4,14)$ & $178(7,3)$ & $4,59(2,95-7,14)^{\star}$ \\
\hline T. de la sangre, $n(\%)$ & $88(4,6)$ & $54(10,2)$ & $142(5,8)$ & $1,05(0,76-1,47)$ \\
\hline T. aparato reproductor, $\mathrm{n}(\%)$ & $8(0,4)$ & $4(0,8)$ & $12(0,5)$ & $1,29(0,39-4,29)$ \\
\hline T. del metabolismo y nutrición, n (\%) & $21(1,1)$ & $2(0,4)$ & $23(1,0)$ & $6,79(1,59-28,92)^{\star}$ \\
\hline T. sistema inmunológico, $\mathrm{n}(\%)$ & $20(1,1)$ & $1(0,2)$ & $21(0,9)$ & $12,93(1,74-96,28)^{\star}$ \\
\hline T. sistema nervioso, n (\%) & $64(3,4)$ & $21(4,0)$ & $85(3,5)$ & $1,97(1,21-3,21)^{\star}$ \\
\hline T. endócrinos, n (\%) & $14(0,7)$ & $3(0,6)$ & $17(0,7)$ & $3,01(0,87-10,50)$ \\
\hline T. gastrointestinales, $\mathrm{n}(\%)$ & $101(5,3)$ & $41(7,7)$ & $142(5,8)$ & $1,59(1,11-2,28)^{\star}$ \\
\hline T. lugar administración, n (\%) & $86(4,5)$ & $10(1,9)$ & $96(4,0)$ & $6,0(3,13-11,52)^{\star}$ \\
\hline T. hepatobiliares, $n(\%)$ & $34(1,8)$ & $21(4,0)$ & $55(2,3)$ & $1,05(0,61-1,80)$ \\
\hline T. musculoesqueléticos, $n(\%)$ & $44(2,3)$ & $24(4,5)$ & $68(2,9)$ & $1,19(0,72-1,94)$ \\
\hline T. oculares, $\mathrm{n}(\%)$ & $38(2,0)$ & $21(4,0)$ & $59(2,4)$ & $1,17(0,69-1,99)$ \\
\hline T. psiquiátricos, n (\%) & $12(0,6)$ & $1(0,2)$ & $13(0,5)$ & $7,76(1,01-59,63)^{\star}$ \\
\hline T. renales y urinarios, $\mathrm{n}(\%)$ & $30(1,6)$ & $2(0,4)$ & $32(1,3)$ & $1,55(1,24-1,94)^{\star}$ \\
\hline T. respiratorios, tórax, mediastino, n (\%) & $48(2,5)$ & $10(1,9)$ & $58(2,4)$ & $3,10(1,57-6,12)^{\star}$ \\
\hline T. vasculares, $\mathrm{n}(\%)$ & $32(1,7)$ & $9(1,7)$ & $41(1,7)$ & $2,30(1,10-4,80)^{\star}$ \\
\hline
\end{tabular}

Abreviaturas: T, trastorno. ${ }^{*} p<0,05$.

\section{Tabla 5. Eventos adversos, riesgo relativo de eventos adversos en tratamiento con agente biológico.}

enfermedad, aspectos genéticos, de respuesta individual o a inmunogenicidad adquirida con el uso de estos agentes. El grupo español analizó los patrones de discontinuación en 10 años y encontró que recientemente la discontinuación en el primer año de tratamiento fue mayor a causa de pérdida de eficacia de los agentes, mientras que la discontinuación por eventos adversos permaneció estable; interpretaron que esto podía deberse a cambios en las guías y prácticas clínicas sobre la introducción, la duración y el cese de los tratamientos con antagonistas del $\mathrm{TNF}^{14}$. Los eventos adversos más frecuentes fueron del grupo “infecciones”, con una amplia ventaja sobre las demás categorías, coincidente con lo informado por los registros internacionales ${ }^{12-14}$; ya dentro de la categoría, las infecciones más comunes fueron las cutáneas y las del tracto respiratorio, también en coincidencia con lo informado en otros registros ${ }^{12-14}$.

A pesar de que se sabe que los tratamientos biológicos presentan mayor riesgo de infecciones comparados con tratamientos no biológicos ${ }^{15}$, los datos deben tomarse con precaución. Para la interpretación de este informe preliminar se deben tomar en cuenta las limitaciones del estudio; principalmente, el posible sesgo en la información de acontecimientos adversos. Probablemente se informen más frecuentemente acontecimientos adversos en pacientes con tratamiento biológico ya que los mismos tienen un seguimiento más estricto que los pacientes en tratamiento no biológico. Asimismo, si bien en el protocolo se preveía el ingreso de un paciente control por cada paciente con tratamiento biológico, no se llegó a la relación deseada, por lo que la proporción de pacientes con tratamientos biológicos supera a la de no biológicos. Se puede pensar acerca de las razones posibles para esta diferencia: la dificultad para identificar pacientes con características similares como así también la probable percepción de los investigadores de que se deben ingresar principalmente casos y no tanto controles por el tipo de registro. Se está trabajando para controlar la calidad de los datos ingresados.

Los agentes biológicos posibilitan un avance en el tratamiento de enfermedades reumáticas, especialmente en aquellos pacientes que no respondían a la medicación no biológica ${ }^{16}$. Estos agentes se han convertido en el tratamiento de elección sobre todo en pacientes con enfermedad activa y grave $e^{16,17}$.

Es esencial avanzar en el estudio de estos tratamientos a largo plazo mediante registros con rigor metodológico. En los últimos años se han implementado mecanismos más estrictos de monitorización para mejorar la calidad de los datos y, por ende, de los informes para, así, obtener los 
mejores resultados en el tratamiento de las enfermedades reumáticas.

\section{Bibliografía}

1. Conti F, Ceccarelli F, Massaro L, Cipriano E, Di Franco M, Alessandri C, et al. Biological therapies in rheumatic diseases. Clin Ter [Internet]. 2013; 164(5):e413-28. Available from: http://www.ncbi. nlm.nih.gov/pubmed/24217844

2. Gómez-Reino JJ, Carmona L, Valverde VR, Mola EM, Montero MD; BIOBADASER Group. Treatment of Rheumatoid Arthritis with Tumor Necrosis Factor Inhibitors May Predispose to Significant Increase in Tuberculosis Risk. Arthritis Rheum Aug 2003; 48(8):2122-2127.

3. Carmona L1, Descalzo MA, Perez-Pampin E, Ruiz-Montesinos D, Erra A, Cobo T, GómezReino JJ; BIOBADASER and EMECAR Groups. All-cause and cause-specific mortality in rheumatoid arthritis are not greater than expected when treated with tumour necrosis factor antagonists. Ann Rheum Dis 2007; 66:880-885

4. Dixon WG1, Watson K, Lunt M, Hyrich KL, Silman AJ, Symmons DP; British Society for Rheumatology Biologics Register. Rates of Serious Infection, Including Site-Specific and Bacterial Intracellular Infection, in Rheumatoid Arthritis Patients Receiving Anti-Tumor Necrosis Factor Therapy. Arthritis Rheum Aug 2006; 154(8):2368-2376.

5. Harrison $\mathrm{MJ}$ et al. Rates of new-onset psoriasis in patients with rheumatoid arthritis receiving antitumour necrosis factor $\alpha$ therapy: results from the British Society for Rheumatology Biologics Register. Ann Rheum Dis 2009; 68:209-215.

6. Dixon WG, Watson KD, et al. Influence of AntiTumor Necrosis Factor Therapy on Cancer Incidence in Patients With rheumatoid Arthritis Who Have Had a Prior Malignancy. Results From the British Society for Rheumatology Biologics Register. Arthr Care \&Research June 2010; 62(6):755-763.

7. Askling J, Baecklund E, Granath F, et al Antitumor necrosis factor therapy in rheumatoid arthritis and risk of malignant lymphomas: relative risks and time trends in Swedish Biologics Register. Ann Rheum Dis 2009; 68:648-653.

8. Strangfeld A, Listing J, Herzer P, Liebhaber A,
Rockwitz K, Richter C, Zink A. Risk of herpes zoster in patients with rheumatoid arthritis treated with anti-TNF $\alpha$ agents. Jama, Feb 18, 2009: 301(7) 737-44.

9. Kvien TK1, Heiberg, Lie E, Kaufmann C, Mikkelsen K, Nordvåg BY, Rødevand E. A Norwegian Register: Prescriptions of DMARDs and biological agents to patients with inflamatory rheumatic diseases. Clin Exp Rheumatol 2005; 23(Suppl 39):S188-S194.

10. Du Pan SM1, Dehler S, Ciurea A, Ziswiler HR, Gabay C, Finckh A, Swiss Clinical Quality Management Physicians. Comparison of drug retention rates and causes of drug discontinuation betwen anti-tumor necrosis factor agents in rheumatoid Arthritis. Arthritis \& Rheumatism may 15, 2009; 61(5):560-568.

11. Greenberg JD1, Kremer JM, Curtis JR, et al. Tumour Necrosis Factor antagonist use and associated risk reduction of cardiovascular events among patients with rheumatoid arthritis. Ann Rheum Dis. 2011 Apr; 70(4):576-82.

12. Titton DC, Silveira IG, Louzada-Junior P, et al. Brazilian Biologic Register: Biobadabrasil implementation process and preliminary results. Rev Bras Reumatol 2011; 51(2):145-160.

13. Descalzo MÁ, Carmona L; Grupo de Estudio BIOBADASER. Biobadaser 2.0: análisis y tendencias en 2009. Reumatol Clin 2010; 6(5):240-243.

14. Gómez-Reino JJ, Rodríguez-Lozano C, Campos-Fernández C, Montoro M, Descalzo MÁ, Carmona L. Change in the discontinuation pattern of tumour necrosis factor antagonists in rheumatoid arthritis over 10 years: data from the Spanish registry BIOBADASER 2.0. Ann Rheum Dis 2012; 71(3):382-5.

15. Ramiro S, Gaujoux-Viala C, Nam JL, Smolen JS, Buch M, Gossec L, et al. Safety of synthetic and biological DMARDs: a systematic literature review informing the 2013 update of the EULAR recommendations for management of rheumatoid arthritis. Ann Rheum Dis [Internet]. 2014; 73(3):529-35.

16. MaX, Xu S. TNF inhibitor therapy for rheumatoid arthritis (Review). Biomed Reports [Internet]. 2013; 1(2):177-84.

17. Huynh D, Kavanaugh A. Psoriatic arthritis: current therapy and future approaches. Rheumatology [Internet]. 2015;54(1):20-8. 